\title{
The antioxidant effect of Brazil nut (Bertholletia excelsa) is influenced by a genetic superoxide- hydrogen peroxide imbalance in healthy humans
}

\begin{abstract}
Some plants are rich in a specific antioxidant that has a glutathione peroxidase -like effect, such as Brazil nut (BN) (Bertholletia excelsa), rich in selenium. This effect could be relevant to human health among people with a genetic superoxide anionhydrogen peroxide (SA-HP) imbalance caused by a single nucleotide polymorphism located in the gene of manganese-dependent superoxide dismutase (Val16Ala-SOD2, rs4880). The AA genotype results in higher SOD2 levels leading to an increase in hydrogen peroxide levels related to a risk of some cancers. Because there evidence suggesting that habitual consumption of fruits and vegetables can minimize this risk, we hypothesized that $\mathrm{BN}$ is beneficial to subjects with genotype AA by decreasing the basal SA-HP imbalance. We performed an analysis of the acute antioxidant effect of one-time ingestion of $10 \mathrm{~g}$ of $\mathrm{BN}$ among healthy adult subjects carrying different SOD2 genotypes. The results showed differential antioxidant responses according to genotypes. Subjects AA showed a reduction in lipoperoxidation and DNA damage, but in these subjects, there was upregulation of thiols and GPX levels at enzymatic and mRNA expression levels. Taken together, our results corroborate the relevance of nutrigenetic effects of ingestion of some plants for modulation of the genetic SA-HP imbalance.
\end{abstract}

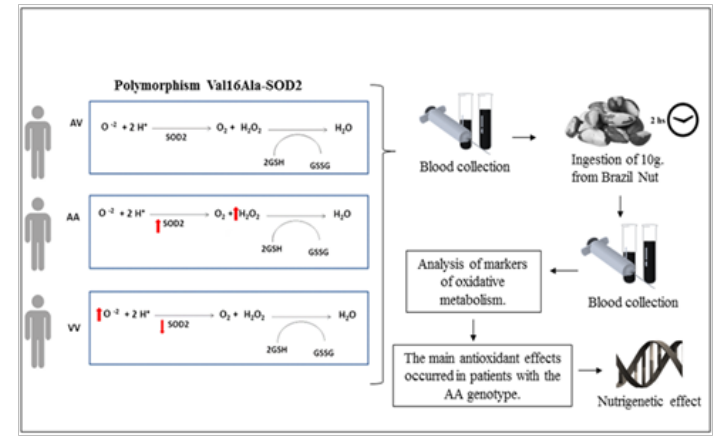

Graphical abstract.

Keywords: nutrigenetic, brazil nut, oxidative stress, lipoperoxidation
Volume 8 Issue 3 - 2018

\author{
Ivana Beatrice Mânica da Cruz,, 1,2,3 Jéssica \\ de Rosso Motta,' Adriano Flesch Lenz,' \\ Thamara Graziela Flores, ${ }^{2}$ Verônica Farina \\ Azzolin, ${ }^{3}$ Maiquidieli Dal Berto, ${ }^{4}$ Alencar \\ Kolinski Machado, ${ }^{5}$ Ednea Aguiar Maia \\ Ribeiro, ${ }^{6}$ Euler Esteves Ribeiro, ${ }^{6}$ Fernanda \\ Barbisan $^{2}$ \\ 'Morphology Department, Federal University of Santa Maria, \\ Brazil \\ ${ }^{2}$ Graduate Program in Gerontology, Federal University of Santa \\ Maria, Brazil \\ ${ }^{3}$ Graduate program of Pharmacology, Federal University of Santa \\ Maria, Brazil \\ ${ }^{4}$ Graduate Program in Pathology, Federal University of Health \\ Sciences of Porto Alegre, Brazil \\ ${ }^{5}$ Franciscan University Center, Brazil \\ ${ }^{6}$ Open University of the Third Age, State University of \\ Amazonas, Brazil
}

Correspondence: Fernanda Barbisan, Graduate Program in Gerontology, Federal University of Santa Maria, Centro de Educação Física e Desportos, Avenida Roraima, 1000 prédio 5I, sala 2032 97।05-900, Santa Maria-RS, Brazil, Tel +55 5532208431,Email fernandabarbisan@gmail.com

Received: February 23, 2018 | Published: May 21, 2018
Abbreviations: BN, Brazil nut; ROS, reactive oxygen species; Se, selenium; GPX, glutathione peroxidase; SNP, single nucleotide polymorphism; SOD, superoxide dismutase; V, valine; A, alanine; HP, hydrogen peroxide; HR, hydroxyl radical; PBMCs, peripheral-blood mononuclear cells; RFLP, restriction fragment length polymorphism; BMI, body mass index; CRP, C-reactive protein; TACO, table of food composition; DCFDA; dichlorofluoresceindiacetate; DCF, dichlorofluorescein; TBARS, thiobarbituric acid reactive substances; $\mathrm{NO}$, nitric oxide

\section{Introduction}

Robust evidence suggests that consumption of fruit and vegetables represents a protective factor against chronic degenerative disorders, such as cancer and cardiovascular diseases, because of the antioxidant properties. ${ }^{1}$ Nonetheless, this antioxidant effect is not universal, in terms of efficacy against reactive oxygen species (ROS). On the other hand, antioxidant compounds can have differential specificity to different ROS types depending on genomic regulation of antioxidant metabolism. ${ }^{2,3}$ This is the case for Brazil nut (BN) Bertholletia excelsa, which is broadly consumed in the world and is known as one of the richest sources of selenium (Se), although the concentration of this chemical element can vary depending on soil conditions. ${ }^{4}$ Because Se is a cofactor of the antioxidant enzyme glutathione peroxidase (GPX), $\mathrm{BN}$ may improve the GPX-like effect, important for modulation of some genetic alterations that cause a basal oxidative imbalance in the cell.

Probably, this is the case for a single nucleotide polymorphism (SNP) located in the gene of manganese-dependent superoxide dismutase (SOD2, rs4880), generically named Val16Ala-SOD2 because it has two alleles ( $\mathrm{T}$ or $\mathrm{C}$ ) that change 16 amino acid 
positions to valine (V) to alanine (A) generating three possible genotypes: VV, AA, and AV (Figure 1). To understand the relevance of this polymorphism, it is important to point out that mitochondria continually produce superoxide anion (SA, as a byproduct of aerobic metabolism), which is a potent ROS. The SOD2 enzyme functions in mitochondria dismutating SA into hydrogen peroxide (HP). After that, HP is converted into water and molecular oxygen by GPX, a selenium-dependent enzyme. ${ }^{5}$

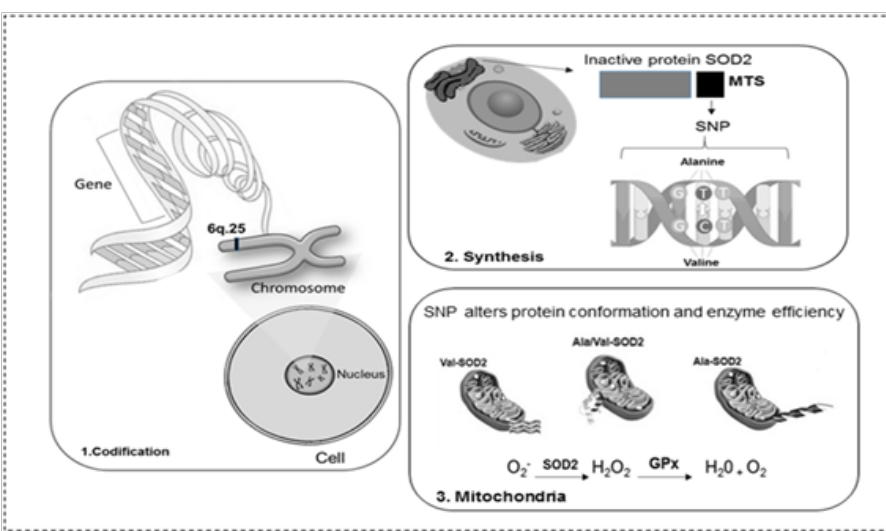

Figure 2 Vall6Ala-SOD2 polymorphism encoding, protein synthesis and enzymatic activation in mitochondria. I. The SOD2 gene is located on chromosome 6 long arm region 25.3. 2. In the cytoplasm the SOD2 protein is synthesized in the rough endoplasmic reticulum, being directed to the mitochondria by the Mitochondrial Target Sequence (MTS). The case is that in the MTS region a single nucleotide polymorphism (SNP) polymorphism occurs, involving the exchange of a single nucleotide, occurring the substitution of a thymine $(T)$ by a cytosine $(C)$ in exon 2 , nucleotide 47 . Such substitution affects codon 16, which encodes amino acid 9, resulting in the replacement of the amino acid valine (GTT) with alanine (GCT). 3. When passing through the internal mitochondrial membrane pores, the MTS peptide segment is cleaved by lysosomes, and the mature protein aggregates in an active form becoming a functional enzyme, however in phenotypic terms, the Ala-SOD2 variant has an $\alpha$ - helix, thus being easily imported into the mitochondria, resulting in increased enzymatic activity. The Val-SOD2 variant has a partial $\beta$-lamina structure, which causes it to be partially retained in the pore of the internal mitochondrial membrane, and therefore the enzymatic activity of this variant can be 30 to $40 \%$ lower than the Ala -SOD2, the Ala /Val-SOD2 variant already has a helical structure with intermediate enzymatic activity.

SOD2 is produced by a nuclear gene located on chromosome 6 , and in this gene, the $\mathrm{V}$ allele produces a $\beta$-sheet protein, whereas the A allele produces an $\alpha$-helical protein. The A-SOD2 protein gets into mitochondria easily, increasing the catalysis of SA conversion to HP. For this reason, it is believed that AA genotype carriers have a more efficient SOD2 enzyme. On the contrary, the $\mathrm{V}$ allele protein stays partially arrested in the mitochondrial membrane, and for this reason, it is thought that VV genotype carriers have a less efficient SOD2 enzyme. ${ }^{6}$ Therefore, the two homozygous genotypes have different SOD2 efficacy that causes an SA-HP imbalance because the VV version of the enzyme cannot dismutate SA efficiently into HP, whereas the enzyme of genotype AA produces higher HP levels than the VV genotype enzyme does. In this regard, AV is considered an "SA-HP balanced genotype"?

This genetic imbalance has been associated with differential oxidative stress in cells and with a strong inflammatory response for the AA-SOD2 genotype and a chronic inflammatory response for the VV-SOD2 genotype. ${ }^{8}$ The AA-SOD2 genotype, or in some cases the A allele, has been described as a risk factor of some cancer types. ${ }^{9} 10$
The main hypothesis to explain this association is related to the fact that the HP excess generated by A-SOD2 does not trigger an increase in GPX levels, and this situation poses a major problem because HP goes to the cytoplasm and reacts with metal ions $(\mathrm{Fe}, \mathrm{Zn})$ producing some dangerous ROS, mainly the hydroxyl radical (HR). There is no endogenous antioxidant control of HR levels, which needs to be catalyzed by nonenzymatic molecules mainly obtained from food. If this control is not efficient, HR could generate extensive DNA damage (and mutations) because HR has high affinity for this biopolymer. ${ }^{11}$

Nonetheless, additional studies suggest that fruits and vegetables may minimize the risk of diseases associated with Val16Ala-SOD2 SNP. ${ }^{12-15}$ Because fruits contain a large number of polyphenols and micronutrients with antioxidant action, two studies involving in vitro analysis were conducted to evaluate the effect of isolated molecules (resveratrol and seleno-L-methionine, SeMet) on oxidative modulation of peripheral-blood mononuclear cells (PBMCs) with different Val16Ala-SOD2 genotypes. ${ }^{16,17}$ The results suggested that, mainly SeMet can exert a major antioxidant effect in both homozygous genotypes by modulation of GPX and of other antioxidant molecules. ${ }^{17}$

According to Schott's ${ }^{17}$ results, we hypothesized that $\mathrm{BN}$ has a beneficial effect on AA genotype subjects by decreasing the basal SA-HP imbalance. To test this hypothesis, we performed an in vivo analysis of an acute antioxidant effect of one-time ingestion of $10 \mathrm{~g}$ of BN Bertholletia excelsa among healthy volunteers with different Val16Ala-SOD2 genotypes.

\section{Materials and methods}

\section{Study design and selection of volunteers carrying different SOD2 genotypes}

An open protocol was performed on healthy volunteers carrying different Val16Ala-SOD2 genotypes who ingested 10g of BN after $12 \mathrm{~h}$ fasting. Analysis of levels of oxidative-metabolism variables was performed and compared between the time point before $\mathrm{BN}$ ingestion and $2 \mathrm{~h}$ after its ingestion.

The study was conducted according to the guidelines laid down in the Declaration of Helsinki, and all procedures were reviewed and approved by the Federal University of Santa Maria Ethics Committee (CAEE number 23081.015838/2011-10). Written informed consent was obtained from all the volunteers. The volunteers were selected from a databank containing 160 subjects enrolled from the university community with ages ranging from 18 to 30 years, who were previously genotyped on Val16Ala-SOD2. It is important to point out that some volunteers participated in prior studies by donating blood samples for in vitro protocols. ${ }^{16-18}$ These subjects were previously SOD2genotyped by the protocol described elsewhere. ${ }^{18}$ Briefly, genomic DNA was isolated from peripheral-blood leukocytes using the DNA Mini Kit Purification (Mo Bio). SOD polymorphism was detected by PCR with restriction fragment length polymorphism (RFLP) analysis. The amplification primers (Gibco Inc, Co.) used for SOD2 genotyping were designed to amplify a 110-base pair (bp) fragment of the human SOD2 gene: 5'-ACCAGCAGGCAGCTGGCGCCGG-3' (sense strand) and 5'-GCGTT GATGTGAGGTTCCAG-3' (antisense strand). The final PCR product $(10 \mu \mathrm{l})$ was digested with Hae III (Gibco. Inc, Co.) producing two bands ( 23 and $85 \mathrm{bp}$ ) visualized on a $4 \%$ agarose gel stained with ethidium bromide (Amersham Biosciences Inc, Co). Genotype detection was made by gel band analysis: VV=100 $\mathrm{bp} ; \mathrm{AA}=23$ and $85 \mathrm{bp}$, and $\mathrm{AV}=23,85$, and $110 \mathrm{bp}$ bands. In the all 
databanks, the $S O D 2$ genotypic frequency was as follows: $\mathrm{AA}=51$ (31.7\%); $\mathrm{VV}=32(20.5 \%)$, and $\mathrm{AV}=77(47.8 \%)$. When a databank was structured, genotype frequencies observed among the volunteers were in Hardy-Weinberg equilibrium.

To carry out the protocol, we chose 6 subjects carrying each genotype (total $n=18,6$ males, 12 females). The inclusion criteria for samples in this protocol were as follows: only subjects without a history of diseases and disorders that could influence the results, e.g., coronary heart disease, stroke, neoplasias, overweight, and obesity (body mass index, BMI $>25 \mathrm{~kg} / \mathrm{m}^{2}$ ), type 2 diabetes, metabolic syndrome, as well as subjects taking any medication regularly. Smokers and pregnant women were also excluded from the analysis. Subjects that had any infection in the last month, or even inflammation, fever, metabolic stress, allergy to oleaginous food, or gastrointestinal disturbances were excluded too. Besides, we excluded subjects that self-reported intensive physical activity in the same period.

Finally, a biochemical analysis of the lipid profile, glycemia, and of some oxidative and inflammatory parameters was conducted among the subjects carrying different Ala16Val-SOD2 genotypes by means of blood sample collection after $12 \mathrm{~h}$ of fasting. In this analysis, 18 persons with different SOD2 genotypes (6 for each genotype) that had similar mean values of these biochemical parameters were finally selected to participate in the experimental protocol. Plasma was used to determine the levels of fasting glucose, total serum cholesterol, and triglyceride concentrations by standard enzymatic methods by means of Ortho-Clinical Diagnostics ${ }^{\circledR}$ reagents on a fully automated analyzer (Vitros 950®dry chemistry system; Johnson \& Johnson, Rochester, NY, USA). High-density lipoprotein cholesterol was measured in the supernatant (plasma) after the precipitation of apolipoprotein B-containing lipoproteins with dextran sulfate and magnesium chloride as described previously. ${ }^{19}$ Low-density lipoprotein cholesterol was estimated with the Friedewald equation. ${ }^{20}$ A high-sensitivity $\mathrm{C}$-reactive protein (hs-CRP) assay was conducted by nephelometry (Dade Behring, Newark, DE, EUA). Biochemical analysis of parameters associated with oxidative metabolism was performed in blood samples collected from the subjects under study. Table 1 presents baseline characteristics of the volunteers included in the intervention analysis.

Table I Baseline characteristics biomarkers of healthy volunteers with different Ala 16Val-SOD2 genotypes

\begin{tabular}{llll}
\hline Variables & AA Mean \pm SD & AV Mean \pm SD & VV Mean \pm SD \\
\hline BMI $\left(\mathrm{Kg} / \mathrm{m}^{2}\right)$ & $22.6 \pm 1.3^{\mathrm{a}}$ & $22.4 \pm 1.3^{\mathrm{a}}$ & $22.5 \pm 1.4^{\mathrm{a}}$ \\
$\begin{array}{l}\text { Cholesterol total } \\
(\mathrm{mg} / \mathrm{dL})\end{array}$ & $168.6 \pm 45.5^{\mathrm{a}}$ & $237.7 \pm 63.4^{\mathrm{a}}$ & $204.8 \pm 58.6^{\mathrm{a}}$ \\
$\mathrm{HDL}-\mathrm{c}(\mathrm{mg} / \mathrm{dL})$ & $48.1 \pm 9.5^{\mathrm{a}}$ & $49.3 \pm 13.4^{\mathrm{a}}$ & $47.0 \pm 13.9^{\mathrm{a}}$ \\
$\mathrm{LDL}-\mathrm{c}(\mathrm{mg} / \mathrm{dL})$ & $85.1 \pm 33.8^{\mathrm{a}}$ & $114.2 \pm 52.4^{\mathrm{a}}$ & $119.1 \pm 55.8^{\mathrm{a}}$ \\
$\begin{array}{l}\text { Triglycerides }(\mathrm{mg} / \\
\mathrm{dL})\end{array}$ & $105.8 \pm 54.3^{\mathrm{a}}$ & $159.9 \pm 74.4^{\mathrm{a}}$ & $118.4 \pm 72.1^{\mathrm{a}}$ \\
Glucose $(\mathrm{mg} / \mathrm{dL})$ & $84.4 \pm 9.4^{\mathrm{a}}$ & $85.1 \pm 8.4^{\mathrm{a}}$ & $101.4 \pm 7.3^{\mathrm{a}}$ \\
\hline
\end{tabular}

SD, standard deviation; Comparison among genotypes was performed by Two way ANOVA analysis of variance followed by Bonferroni post hoc test. *, statistical differences where $\mathrm{p}<0.05$.

During this first selection, volunteers were contacted and invited to participate in the protocol 2 weeks before BN consumption and data collection. The experimental protocol was explained in detail to all the volunteers; they were instructed to not consume antioxidant foods (vegetables, salads, fruits, and juices and foods rich in selenium such as eggs, egg yolk, cereals, and organ meats) throughout $24 \mathrm{~h}$ of the blood sampling. In Brazil, the main daily meal takes place at noon; for this reason, the volunteers were advised to consume this meal in the 15 days immediately before the experiment at the university cafeteria, which provided a nutritionally balanced meal.

\section{The general intervention protocol}

When the volunteers arrived in the Biogenomic Lab located on the UFSM campus, 12 basal fasting blood samples were collected by venipuncture using top Vacutainers (tubes with heparin). Immediately after the first blood sample collection, the volunteers ingested $10 \mathrm{~g}$ of BN with $150 \mathrm{~mL}$ of filtered cold water and remained at rest for $2 \mathrm{~h}$ when the second blood collection was made. The $2 \mathrm{~h}$ interval was chosen to avoid the ingestion of other foods or liquids that could influence the results. In this case, a longer waiting period would cause hypoglycemia and hypotension, as well as metabolic stress causing discomfort to the volunteers and some potential alteration of oxidative metabolism.

\section{BN: origin, nutritional characteristics, and preparation}

The BNs consumed by volunteers were obtained in Manaus city, Amazonas state, and had the following estimated bromatological characteristics per $10 \mathrm{~g}$ as described in the Brazilian Table of Food Composition $(\mathrm{TACO})^{21}$ calories $=67 \mathrm{kcal}$; total fat $=6.7 \mathrm{~g}$; saturated fat $=1.9 \mathrm{~g}$; polyunsaturated fat $=2.5 \mathrm{~g}$ monounsaturated fat $=2.5 \mathrm{~g}$; total carbohydrates $=1.0 \mathrm{~g}$; total protein $=1.4 \mathrm{~g}$; dietary fiber $=0.7 \mathrm{~g}$; sodium $=0.4 \mathrm{mg} ; \quad$ potassium $=66.7 \mathrm{mg} ; \quad$ calcium $=1.8 \%$; iron $=1.4 \%$. To ensure that each volunteer ingested a similar quantity of $\mathrm{BN}$ $(10 \mathrm{~g})$, BNs were stung, weighed, and stored in a plastic bag until consumption. This procedure was done $30 \mathrm{~min}$ before the beginning of the blood collection to avoid oxidation of $\mathrm{BN}$. This procedure was done aseptically.

\section{ROS production assay}

ROS production was determined using a nonfluorescent cellpermeating compound called 2-7-dichlorofluoresceindiacetate (DCFDA).$^{18}$ In this assay, DCFDA is hydrolyzed by intracellular esterases to DCFH, which is trapped within the cell. This nonfluorescent molecule is then oxidized to fluorescent dichlorofluorescein (DCF) by cellular oxidative factors. After the designated treatment period, the plasma blood was treated with DCFDA $(10 \mu \mathrm{M})$ for $60 \mathrm{~min}$ at $37^{\circ} \mathrm{C}$. The fluorescence was measured via excitation at $488 \mathrm{~nm}$ and emission at $525 \mathrm{~nm}$ on a microplate reader (SpectraMax M2e, Molecular Devices, Austria).

\section{Free double-stranded DNA (dsDNA) assay}

Prior evidence revealed that higher levels of dsDNA in the plasma are associated with inflammatory and oxidative status due an increase in mortality of blood white cells, and Cadoná et al.,22 showed that pro-oxidant and antioxidant conditions can quickly change dsDNA concentrations because in a pro-oxidant environment, there could be an increase in the number of dead cells, whereas in an antioxidant environment, a delay in cell mortality may occur. Therefore, quantification of free plasma dsDNA was performed according to the protocol of Costa et al. ${ }^{23}$ Briefly, $10 \mu 1$ samples of plasma obtained from different volunteers were added into wells of a 96-well black plate together with reagents from the Quant-iTTM PicoGreen ${ }^{\circledR}$ 
Kit purchased from Invitrogen (Eugene, OR, USA) and diluted in TE buffer $(10 \mathrm{~mm}$ Tris-HCl, $1 \mathrm{~mm}$ EDTA, $\mathrm{pH} 7.5)$ with reagents obtained from Sigma-Aldrich (St. Louis, MO, USA). Then, $90 \mu 1$ of the PicoGreen dye diluted 1:200 in Tris-HCl EDTA (TE) buffer was added to the microplate to attain a final volume of $100 \mu 1$ per well. Following incubation in the dark for $5 \mathrm{~min}$ at room temperature, the fluorescent signals of the samples were measured at $480 \mathrm{~nm}$ excitation and $520 \mathrm{~nm}$ emissions on the microplate reader (SpectraMax M2e, Molecular Devices, Austria)

\section{Quantification of protein carbonylation, thiol groups, and lipoperoxidation}

Protein carbonyls were measured according to the method described elsewhere. ${ }^{24}$ Differential colorimetric formation was measured at $370 \mathrm{~nm}$. Results were expressed in nanomoles of carbonyl groups per milligram of protein. Thiol groups were colorimetrically determined as described by Ellman. ${ }^{25}$ Both measurements were conducted on a microplate reader (SpectraMax M2e, Molecular Devices, Austria). Lipoperoxidation was spectrophotometrically estimated by the formation of thiobarbituric acid reactive substances (TBARS), as described by Jentzsch et al. ${ }^{26}$

\section{Activity of antioxidant enzymes}

Analysis of treatment effects on the endogenous antioxidant enzymes SOD, CAT, and GPX was performed using commercial kits specific to each enzyme (Sigma-Aldrich).

\section{Statistical analyses}

These analyses were performed in the Graph Pad Prism software, version 5. General comparison between samples before and after BN ingestion was performed by Student's paired $t$ test. Comparison of different Val16Ala-SOD2 genotypes in terms of oxidative variables evaluated here was performed by two-way analysis of variance followed by the Bonferroni post hoc test. Data with $\mathrm{p}$ values $<0.05$ were considered statistically significant

\section{Results}

Basal oxidative-metabolism variables were determined and are shown in Table 2. As for pro-oxidant molecules, VV genotype subjects had higher lipoperoxidation and protein carbonylation levels than A allele subjects, whereas A allele subjects showed higher dsDNA plasma levels than subjects of the VV genotype did. Thiols, nonenzymatic antioxidant molecules, were also influenced by SOD2 genotypes, because VV genotype subjects showed higher levels of this molecule than subjects with the AA genotype did. In addition, antioxidant-enzyme levels were strongly influenced by the SOD2 polymorphism. Total SOD levels were higher in volunteers with the AA genotype than in the other subjects. On the contrary, GPX levels were higher in volunteers with the VV genotype than in A allele subjects. CAT showed some differential levels according to the three genotypes: genotype AA was associated with higher levels of this enzyme followed by the VV genotype, whereas heterozygous subjects (AV) showed lower CAT levels.

A general comparison of oxidative-metabolism variables without considering the SOD2 SNP polymorphism revealed that $2 \mathrm{~h}$ after BN ingestion, SOD, GPX, ROS, protein carbonylation, and dsDNA levels did not change significantly when compared to fasting values. By contrast, CAT, total polyphenol levels, and thiols showed upregulation relative to fasting values. Lipoperoxidation evaluated by the TBARS assay was lower when compared to fasting values (Table 3 ).

Table 2 Comparison among basal oxidative metabolism variables of healthy volunteers carrying different Vall6Ala-SOD2 genotypes

\begin{tabular}{|c|c|c|c|c|}
\hline Variables & $\begin{array}{l}\text { AA } \\
\text { Mean } \pm S D\end{array}$ & $\begin{array}{l}\text { AV } \\
\text { Mean } \pm S D\end{array}$ & $\begin{array}{l}\text { VV } \\
\text { Mean } \pm S D\end{array}$ & $\mathbf{p}$ \\
\hline $\begin{array}{l}\text { Protein carbonyl } \\
\text { (nmol/mg protein) }\end{array}$ & $1.6 \pm 0.5^{\mathrm{a}}$ & $1.7 \pm 0.6^{\mathrm{a}}$ & $2.3 \pm 0.2^{b}$ & 0.02 \\
\hline TBARS (nmol/mL) & $5.3 \pm 2.4^{a}$ & $5.6 \pm 2.9^{a}$ & $8.8 \pm 3.8^{a}$ & 0.03 \\
\hline dsDNA (pg/mL) & $38.5 \pm 4.4^{a}$ & $40.3 \pm 2.4^{a}$ & $33.1 \pm 3.1^{b}$ & 0.04 \\
\hline $\begin{array}{l}\text { ROS (DCF nmol/ } \\
\mathrm{mL} \text { ) }\end{array}$ & $48.7 \pm 3.2^{\mathrm{a}}$ & $50.1 \pm 2.0^{\mathrm{a}}$ & $51.4 \pm 3.7^{\mathrm{a}}$ & 0.54 \\
\hline $\begin{array}{l}\text { Polyphenols (mg/ } \\
\mathrm{mL} \text { ) }\end{array}$ & $3.9 \pm 1.0^{\mathrm{a}}$ & $4.5 \pm 0.5^{\mathrm{a}}$ & $4.4 \pm 1.2^{\mathrm{a}}$ & 0.48 \\
\hline $\begin{array}{l}\text { Thiol Groups } \\
\text { (mM/mL plasma) }\end{array}$ & $10.5 \pm 3.4^{\mathrm{a}}$ & $16.6 \pm 1.8^{b}$ & $22.5 \pm 3.5^{c}$ & $<0.001$ \\
\hline $\begin{array}{l}\text { SOD (U SOD/mg } \\
\text { protein) }\end{array}$ & $3.7 \pm 0 . I^{a}$ & $2.7 \pm 0 . I^{b}$ & $2.6 \pm 0.1^{b}$ & 0.01 \\
\hline $\begin{array}{l}\text { GPX-I ( } \mu \mathrm{mol} \text { of } \\
\text { oxidized NADPH/ } \\
\mathrm{min} / \mathrm{mg} / \mathrm{protein})\end{array}$ & $1.2 \pm 0.2^{\mathrm{a}}$ & $1.3 \pm 0.2^{\mathrm{a}}$ & $1.8 \pm 0.1^{b}$ & 0.03 \\
\hline $\begin{array}{l}\mathrm{CAT}(\mathrm{nmol} \text { of } \\
\mathrm{H}_{2} \mathrm{O}_{2} / \mathrm{min} / \mathrm{mg} \\
\text { protein) }\end{array}$ & $1.9 \pm 0.3^{\mathrm{a}}$ & $1.0 \pm 0.1^{\mathrm{b}}$ & $1.3 \pm 0.1^{c}$ & 0.01 \\
\hline
\end{tabular}

SD, standard deviation; Comparison among genotypes was performed by Two way ANOVA analysis of variance followed by Bonferroni post hoc test. Different letters indicate significant differences $(p<0.01)$ among genotype in each variables analyzed here.

Table 3 General modulation of fasting oxidative metabolism variables after $2 \mathrm{~h}$ Brazilian nut ingestion

\begin{tabular}{lll}
\hline Variables (\% fasting values) & Mean \pm SD & $\mathbf{p}$ \\
\hline Protein carbonyl & $98.5 \pm 12.2$ & 0.63 \\
TBARS & $92.3 \pm 3.8$ & 0.02 \\
dsDNA & $96.6 \pm 4.2$ & 0.07 \\
ROS & $96.6 \pm 6.1$ & 0.08 \\
Polyphenols & $113.6 \pm 4.2$ & $>0.01$ \\
Thiol Groups & $121.6 \pm 5$ & $>0.01$ \\
SOD & $102.7 \pm 3.18$ & 0.48 \\
GPX & $107.3 \pm 13.2$ & 0.4 \\
CAT & $132.3 \pm 10.7$ & $>0.001$ \\
\hline
\end{tabular}

$\mathrm{SD}$, standard deviation; Statistical comparison between fasting and $2 \mathrm{~h}$ Brazilian nut ingestion was performed by Student $\mathrm{t}$ test and results are presented as $\%$ of fasting control values.

The effect of the Val16Ala-SOD2 SNP on oxidative-metabolism modulation by $\mathrm{BN}$ ingestion was assessed next. In relation to prooxidant markers, BN ingestion did not affect ROS and protein carbonylation levels in the volunteers carrying different SOD2 genotypes. Nevertheless, a lowering effect on TBARS and plasma dsDNA levels was observed in AA genotype subjects after BN ingestion, whereas this result was not obtained in subjects with the $\mathrm{V}$ allele (Figure 2). 
As for antioxidant markers (Figure 3), thiol levels increased significantly only in A allele subjects when compared to fasting levels. In this case, genotype AA was associated with higher thiol levels than genotype AV was. On the other hand, the increase in polyphenol levels after $\mathrm{BN}$ ingestion was similar among the three genotypes (Figure 4A). BN ingestion did not affect basal SOD levels in subjects carrying different SOD2 genotypes. Although the GPX enzyme level was higher in subjects with genotype AA after BN ingestion, this effect was not observed in subjects carrying the $\mathrm{V}$ allele. A notable increase of CAT levels was observed in subjects with genotype AV after BN ingestion. Subjects with genotype VV also revealed an increase in the levels of this enzyme, but the effect was smaller than that in the $\mathrm{AV}$ genotype subjects. On the contrary, in AA genotype subjects, the levels of the CAT enzyme were not affected by BN ingestion (Figure 4B).

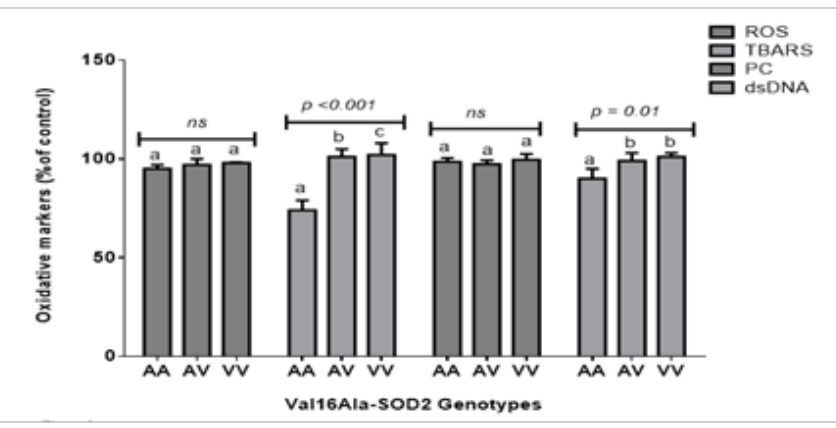

Figure 3 Oxidative Markers- Statistical comparison between fasting and $2 \mathrm{~h}$ Brazilian nut ingestion was performed by Student $\mathrm{t}$ test and results are presented as $\%$ of fasting control values.
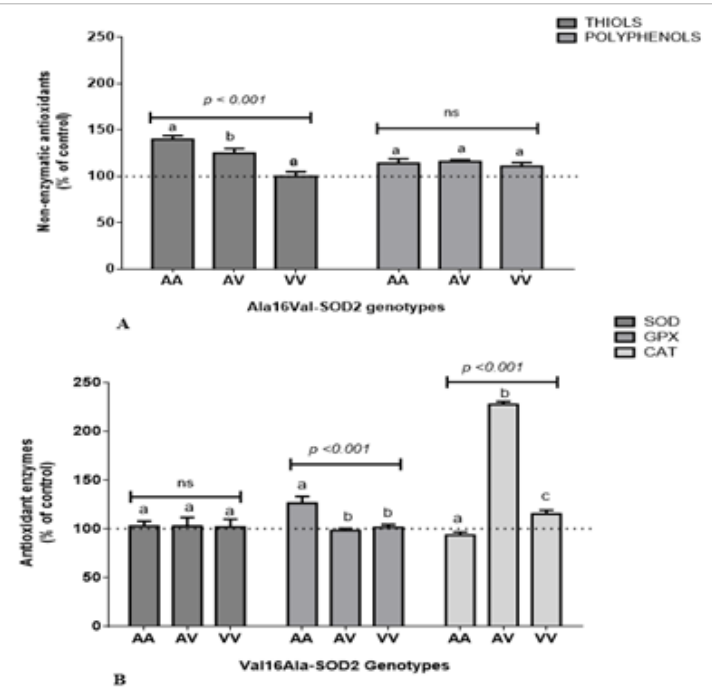

Figure 4 Antioxidant markers A- Non-enzymatic antioxidants. B-Antioxidant enzymes- Statistical comparison between fasting and $2 \mathrm{~h}$ Brazilian nut ingestion was performed by Student $t$ test and results are presented as \% of fasting control values.

\section{Discussion}

The present study describes the acute effect of $\mathrm{BN}$ ingestion among healthy volunteers carrying different Val16Ala-SOD2 genotypes. The antioxidant $\mathrm{BN}$ effects were strongly influenced by a genetic SA-HP imbalance. Before we discuss the nutrigenetic effects observed here, it is important to point out that, to the best of our knowledge, the acute effect of $\mathrm{BN}$ ingestion on blood oxidative markers has not been previously reported in the literature. In fact, most of existing studies deal with other biological properties of BN, such as anti-inflammatory and hypolipemiant action on healthy volunteer ${ }^{27}$ and an antioxidant effect on obese women ${ }^{28}$ and hemodialysis patients. ${ }^{29}$

In our study, BN ingestion significantly increased total blood polyphenol levels, independently of SOD2 SNPs. This result could be expected because $\mathrm{BN}$ in its nutritional matrix contains known polyphenol molecules such as gallic acid, protocatechuic acid, $(+)$-catechin, and p-coumaric acid. ${ }^{30} \mathrm{BN}$ also contains other lignan polyphenol molecules, such as matairesinol and secoisolariciresinol. ${ }^{31}$ Because total polyphenol levels increased in a similar way among the three genotypes, it seems that other differences related to BN effects on oxidative metabolism among the volunteers are probably not related to polyphenol absorption.

In contrast to total polyphenol levels, other oxidative metabolic variables analyzed here were directly affected by SOD2 SNPs. Given that homozygous genotypes showed a basal SA-HP imbalance, we believe that initially, it would be convenient to discuss our results by considering each genotype separately.

It is important to point out that the $\mathrm{V}$ allele also causes SAHP imbalance, in particular, VV-SOD2 genotypes have been associated with the risk of some metabolic disorders, such as hypercholesterolemia, obesity, and cardiovascular diseases ${ }^{32-36}$ and with type 2 diabetes mellitus complications. ${ }^{37}$ The explanation for the increase in the susceptibility to these disorders in the VV genotype is that VV genotype cells maintain high basal SA levels that quickly react with nitric oxide (NO) producing extensive alterations in the cells because lipoperoxidation-derived protein carbonylation generates other ROS types. ${ }^{38}$ Nevertheless, the imbalance associated with the VV genotype is chemically different from that associated with genotype AA because it generates higher SA levels than HP levels.

As mentioned above, the AA genotype is strongly associated with elevated HP levels because a greater quantity of SOD2 gets into mitochondria. In contrast, due to a noncompensatory GPX action, it seems that AA genotype subjects have higher HP basal levels than subjects with genotype VV or AV. This phenomenon is the causal mechanism postulated to explain epidemiological results that suggest that the A allele increases the risk of some cancers. ${ }^{11,38,39}$ We believe that our results support this hypothesis, given that we observed higher fasting CAT levels in AA genotype subjects than in subjects with genotype $\mathrm{AV}$ or VV. This is an indirect HP evaluation, which may be valid because CAT is a crucial enzyme protecting the cell against high HP levels and works mainly in peroxisomes and the cytoplasm. Total levels of the SOD enzyme were also higher in AA genotype subjects than in subjects with the $\mathrm{V}$ allele, probably owing to higher active SOD2 concentrations in AA genotype subjects, as previously described by Shimoda-Matsubayashi et al. ${ }^{6}$

When AA genotype subjects ingested BN, we observed substantial elevation of thiol levels. This effect was weaker in AV genotype subjects, whereas subjects with genotype VV did not show changes in thiol levels after BN ingestion. It is relevant to point out that thiols are molecules that act as antioxidants, stabilizing free radicals by 
accepting their unpaired electron. Thiols are directly related to GSH and GPX function for maintenance of the reduction-oxidation (redox) balance within body tissues. The AA genotype is directly associated with greater metabolic HP production due to higher SOD2 efficiency Jones. ${ }^{40}$

Moreover, AA genotype subjects did not manifest alterations in the SOD and CAT levels after BN ingestion, but we observed a significant increase in GPX activity. GPX is an antioxidant enzyme responsible for catalysis of HP conversion into water in mitochondria. These results are plausible because GPX is a Se-dependent enzyme. Selenium ( $\mathrm{Se}$ ) is an essential micronutrient for humans, acting as a component of noncanonical amino acids: selenocysteine (Se-Cys) and selenomethionine (Se-Met). Supplementation of tissue culture media or animal or human diets with moderate levels of Se compounds may prevent formation of DNA adducts, DNA or chromosome breakage, and chromosome gain or loss. Protective effects on mitochondrial DNA have also been shown, as is the case for the effects on telomere length and function. Some of the effects of Se compounds on gene expression may be related to modulation of DNA methylation or inhibition of histone deacetylation. ${ }^{41}$ It is important to highlight that, in our study, volunteers received $10 \mathrm{~g}$ of $\mathrm{BN}$, which approximately corresponds to the recommended daily allowance for $\mathrm{Se}^{42}$

Under basal conditions, subjects with the AA or AV genotype showed higher dsDNA levels than VV genotype subjects did. The dsDNA quantification has been used to evaluate inflammatory status of some chronic and infectious diseases, ${ }^{43-45}$ and especially in sperm cells, high dsDNA levels have been associated with oxidative stress markers and infertility. ${ }^{23,46}$ In one study reported by Costa et al., ${ }^{47}$ the authors showed that sperm carrying homozygous SOD2 genotypes (AA and VV) have higher dsDNA levels than sperm with the AV genotype. Nonetheless, in our blood samples, basal dsDNA concentration was found to be higher in AA genotype subjects relative to $\mathrm{VV}$ genotype subjects, but only in AA genotype volunteers, did we notice a drop in the levels of this marker after $\mathrm{BN}$ ingestion. Moreover, BN ingestion by AA genotype volunteers had a lowering effect on lipoperoxidation and dsDNA levels, thereby indicating a direct acute antioxidant effect. This effect is consistent with the data of Schott et al., ${ }^{17}$ who conducted an in vitro study testing the SeMet effects on PBMCs of various Val16Ala-SOD2 genotypes. Those authors observed a decrease in TBARS levels after SeMet exposure of AA genotype PBMCs.

Taken together, these results suggest that in subjects with the AA genotype, BN ingestion helped to balance the endogenous oxidative metabolism by increasing thiol levels and GPX levels and by decreasing lipoperoxidation and cellular fragmentation, which are relevant oxidative markers.

In relation to the VV genotype under basal conditions, the subjects carrying this genotype showed higher levels of lipoperoxidationderived protein carbonylation and ROS, but also higher levels of thiols and the GPX enzyme than those in subjects with genotype AA. As mentioned above, the VV genotype is related to higher SA levels because of lower efficiency of the SOD2 enzyme. This genotype's association with metabolic diseases is based on the hypothesis that excess SA reacts with NO thereby producing key nitrosative molecules such as peroxynitrite, that are responsible for increased lipoperoxidation, protein carbonylation, and other oxidative damage to the cell. ${ }^{9}$ Therefore, the elevated levels of basal oxidative markers under study support this hypothesis. Nevertheless, when VV genotype subjects ingested $\mathrm{BN}$, they did not show an increase in thiols or in SOD and GPX levels, but manifested slight upregulation of the CAT enzyme. Any oxidative markers tested here were acutely affected by the $\mathrm{BN}$ ingestion in VV genotype subjects.

In general, AV genotype subjects had similar basal levels of some oxidative-metabolism variables relative to AA genotype subjects, e.g., protein carbonylation, lipoperoxidation, and GPX activity. Only SOD activity was similar to that in subjects with the VV genotype, whereas thiol and CAT levels showed intermediary levels between homozygous subjects. Nonetheless, the BN effect was not as pronounced as that observed in AA genotype subjects. In fact, levels of thiols revealed intermediary elevation when compared to AA genotype subjects. On the other hand, BN induced higher CAT levels in heterozygous subjects. Additionally, strong up regulation of this enzyme was reported by Schott et al., ${ }^{17}$ when AV genotype PBMCs was exposed to SeMet. This similarity suggests that some Se effects may explain our BN effects. Unfortunately, at present, it is not possible to offer a reasonable explanation of this BN action on CAT levels for the AV genotype.

\section{Conclusion}

In summary, our results show nutrigenetic BN effects helping people with the AA genotype to decrease the genetically caused HP levels. Because this study has some important limitations including the low number of volunteers, analysis of few oxidative-metabolism parameters, and a protocol that was focused only on the acute BN effect, additional studies are needed to clarify the BN effects on other biochemical pathways. Moreover, we cannot ignore the fact that BN's antioxidant effects may manifest themselves $2 \mathrm{~h}$ after the first ingestion in all volunteers. For this reason, considering the nutritional properties of $\mathrm{BN}$ and that most people did not know their genetic profile regarding Val16Ala-SOD SNP, habitual consumption of BN may help to decrease the cancer risk in persons with the AA genotype.

\section{Acknowledgments}

This work was performed with financial support (grants and fellowships) from Brazilian research agencies: FAPEAM, FAPERGS and $\mathrm{CNPq}$.

\section{Conflict of interest}

Authors declare no conflict of interest.

\section{References}

1. Griffiths K, Aggarwal BB, Singh RB, et al. Food Antioxidants and Their Anti-Inflammatory Properties: A Potential Role in Cardiovascular Diseases and Cancer Prevention. Diseases. 2016;4(3):1-15.

2. Kaput J, Perlina A, Hatipoglu B, et al. Nutrigenomics: concepts and applications to pharmacogenomics and clinical medicine. Pharmacogenomics. 2007;8(4):369-390.

3. Celis-Morales C, Lara J, Mathers JC. Personalising nutritional guidance for more effective behaviour change. The Proceedings of the Nutrition Society. 2015;74(2):130-138.

4. Silva JEC, Wadt LHO, Silva KE, et al. Natural variation of selenium in Brazil nuts and soils from the Amazon region. Chemosphere. 2017;188:650-658. 
5. Indo HP, Yen HC, Nakanishi I, et al. A mitochondrial superoxide theory for oxidative stress diseases and aging. J Clin Biochem Nutr. 2015;56(1):1-7.

6. Shimoda-Matsubayashi S, Matsumine H, Kobayashi T, et al. Structural dimorphism in the mitochondrial targeting sequence in the human manganese superoxide dismutase gene. A predictive evidence for conformational change to influence mitochondrial transport and a study of allelic association in Parkinson's disease. Biochem Biophys Res Commun. 1996;13:561-565.

7. Sutton A, Khoury H, Prip-Buus C, et al. The Ala16Val genetic dimorphism modulates the import of human manganese superoxide dismutase into rat liver mitochondria. Pharmacogenetics. 2003;13:145-157.

8. Barbisan F, Azzolin VF, Ribeiro EE, et al. The In Vitro Influence of a Genetic Superoxide-Hydrogen Peroxide Imbalance on Immunosenescence. Rejuvenation Res. 2017;20(4):334-345.

9. Bresciani G, González-Gallego J, da Cruz IBM, et al. The Ala16Val MnSOD gene polymorphism modulates oxidative response to exercise. Clin Biochem. 2013;46(4-5):335-340.

10. Kang SW. Superoxide dismutase 2 gene and cancer risk: evidence from an updated meta-analysis. Int J Clin Exp Med. 2015;8(9):14647-14655.

11. Li X, Shen M, Cai H, et al. Association between manganese superoxide dismutase (MnSOD) polymorphism and prostate cancer susceptibility: a meta-analysis. Int J Biol Markers. 2016;31(4):422-430.

12. Ambrosone CB, Freudenheim JL, Thompson PA, et al. Manganese superoxide dismutase (MnSOD) genetic polymorphisms, dietary antioxidants and risk of breast cancer. Cancer Research. 1999;59(3):602606.

13. Cai Q, Shu XO, Wen W, et al. Genetic polymorphism in the manganese superoxide dismutase gene, antioxidant intake, and breast cancer risk: results from the Shanghai Breast Cancer Study. Breast Cancer Research. 2004;6(6):47-55.

14. Choi JY, Neuhouser ML, Barnett MJ, et al. Iron intake, oxidative stressrelated genes (MnSOD and MPO) and prostate cancer risk in CARET cohort. Carcinogenesis. 2008;29(5):964-970.

15. Kakkoura MG, Demetriou CA, Loizidou MA. MnSOD and CAT polymorphisms modulate the effect of the Mediterranean diet on breast cancerrisk among Greek-Cypriot women. Eur J Nutr. 2016;55(4):15351540 .

16. Capeleto D, Barbisan F, Azzolin V, et al. The anti-inflammatory effects of resveratrol on human peripheral blood mononuclear cells are influenced by a superoxide dismutase 2 gene polymorphism. Biogerontology. 2015;16(5):621-630.

17. Schott KL, Assmann CE, Barbisan F, Azzolin, et al. Superoxide-hydrogen peroxide genetic imbalance modulates differentially the oxidative metabolism on human peripheral blood mononuclear cells exposed to seleno-L-methionine. Chem Biol Interact. 2017;273:1-44.

18. Barbisan F, Motta R, Trott A, et al. Methotrexate-related response on human peripheral blood mononuclear cells be modulated by the Ala16ValSOD2 gene polymorphism. PLoS One. 2014;9(10):1-11.

19. Bachorik PS, Albers JJ. Precipitation methods for quantification of lipoproteins. Methods Enzymol. 1986;129:78-100.

20. Friedewald WT, Levy RI, Fredrickson DS. Estimation of the concentration of low-density lipoprotein cholesterol in plasma, without use of the preparative ultracentrifuge. Clin Chem. 1972;18(6):499-502.

21. Galeazzi MAM, Lima DM, Colugnati FAB, et al. Sampling Plan for the Brazilian TACO Project. Journal of Food Composition and Analysis. 2002;15:499-505.
22. Cadoná FC, Manica-Cattani MF, Machado AK, et al. Genomodifier capacity assay: a non-cell test using dsDNA molecules to evaluate the genotoxic/ genoprotective properties of chemical compounds. Analytical Methods. 2014;6(21):8559-8568.

23. Costa F, Barbisan F, Assmann CE, et al. Seminal cell-free DNA levels measured by PicoGreen fluorochrome are associated with sperm fertility criteria. Zygote. 2017;25(2):111-119.

24. Morabito F, Cristani M, Saija A, et al. Lipid peroxidation and protein oxidation in patients affected by Hodgkin's lymphoma. Mediators Inflamm. 2004;13(5-6):381-383.

25. Ellman GL. Tissue sulfhydryl groups. Arch Biochem Biophys. 1959;82(1):70-77.

26. Jentzsch AM, Bachmann H, Furst $P$, et al. Improved analysis of malondialdehyde in human body fluids. Free Radic Biol Med. $1983 ; 20(2): 251-256$.

27. Colpo E, Vilanova CD, Brenner Reetz LG, et al. A single consumption of high amounts of the Brazil nuts improves lipid profile of healthy volunteers. Journal of Nutrition and Metabolism. 2013;1:1-7.

28. Cominetti C, de Bortoli MC, Garrido AB, et al. Brazilian nut consumption improves selenium status and glutathione peroxidase activity and reduces atherogenic risk in obese women. Nutr Res. 2012;32(6):403-407.

29. Stockler-Pinto MB, Mafra D, Farage NE, et al. Effect of Brazil nut supplementation on the blood levels of selenium and glutathione peroxidase in hemodialysis patients. Nutrition. 2010;26(11-12):10651069.

30. Gomes S, Torres AG. Optimized extraction of polyphenolic antioxidant compounds from Brazil nut (Bertholletia excelsa) cake and evaluation of the polyphenol profile by HPLC. J Sci Food Agric. 2016;96(8):28052814 .

31. Gunter GC, Kuhnle CDA, Sue MA, et al. Phytoestrogen Content of Beverages, Nuts, Seeds, and Oils. J Agric Food Chem. 2008;56(16):73117315 .

32. Montano MA, Barrio Lera JP, Gottlieb MG, et al. Association between manganese superoxide dismutase (MnSOD) gene polymorphism and elderly obesity. Mol Cell Biochem. 2009;328(1-2):33-40.

33. Duarte MM, Moresco RN, Duarte T, et al. Oxidative stress in hypercholesterolemia and its association with Ala16Val superoxide dismutase gene polymorphism. Clin Biochem. 2010;43(13-14):11181123 .

34. Chen $\mathrm{H}, \mathrm{Yu} \mathrm{M}, \mathrm{Li} \mathrm{M}$, et al. Polymorphic variations in manganese superoxide dismutase (MnSOD), glutathione peroxidase-1 (GPX1), and catalase (CAT) contribute to elevated plasma triglyceride levels in Chinese patients with type 2 diabetes or diabetic cardiovascular disease. Mol Cell Biochem. 2012;363(1-2):85-91.

35. Souiden Y, Mallouli H, Meskhi S, et al. MnSOD and GPx1 polymorphism relationship with coronary heart disease risk and severity. Biol Res. 2016;49:22.

36. Flores AE, Pascotini ET, Kegler A, et al. ALA16VAL MnSOD gene polymorphism and stroke: Association with dyslipidemia and glucose levels. Gene. 2017;627:57-62.

37. Pourvali K, Abbasi M, Mottaghi A. Role of Superoxide Dismutase 2 Gene Ala16Val Polymorphism and Total Antioxidant Capacity in Diabetes and its Complications. Avicenna J Med Biotechnology. 2016;8(2):48-56.

38. Bresciani G, da Cruz IB, González-Gallego J. Manganese superoxide dismutase and oxidative stress modulation. Adv Clin Chem. 2015;68:87130. 
39. Wanga S, Wang F, Shia $X$, et al. Association between manganese superoxide dismutase (MnSOD) Val-9Alapolymorphism and cancer risk -A meta-analysis. European Journal Cancer. 2009;45:2874-2881.

40. Jones DA, Prior SL, Tang TS, et al. Association between the rs4880 superoxide dismutase $2(\mathrm{C}>\mathrm{T})$ gene variant and coronary heart disease in diabetes mellitus. Diabetes Res Clin Pract. 2010;90(2):196-201.

41. Rayman MP, Infante HG, Sargent M. Food-chain selenium and human health: spotlight on speciation. Br J Nutr. 2008;100(2):238-253.

42. Lemire A, Fillion MA, Barbosa F, et al. Elevated levels of selenium in the typical diet of Amazonian riverside populations Mélanie. Sci Total Environ. 2010;408(19):4076-4084.

43. Cichota LC, Bochi GV, Tatsch E, et al. Circulating double-stranded DNA in plasma of hemodialysis patients and its association with iron stores. Clin Lab. 2015;61(8):985-990.
44. Szeto HH. First-in-class cardiolipin-protective compound as a therapeutic agent to restore mitochondrial bioenergetics. Br J Pharmacol. 2014;171(8):2029-2050.

45. Ulrich BA, Paweletz CP. Cell-Free DNA in Oncology: Gearing up for Clinic. Ann Lab Med. 2017;38(1):1-8.

46. Sabeti P, Pourmasumi S, Rahiminia T, et al. Etiologies of sperm oxidative stress. Int J Reprod Biomed (Yazd). 2016;14(4):231-240.

47. Costa F, Barbisan F, Assmann CE, et al. Influence of Val16Ala-SOD2 polymorphism on sperm quality parameters. Hum Fertil (Camb). 2017;1:1-8 